\title{
Réflexions éthiques et démarche palliative intégrée dans les réanimations pédiatriques françaises en 2017
}

\section{Ethical Thinking and Palliative Approach Integrated at French Paediatric Intensive Care Unit in 2017}

\author{
L. de Saint Blanquat · M.-L. Viallard \\ Reçu le 4 septembre 2017; accepté le 8 juillet 2018 \\ (C) SRLF et Lavoisier SAS 2018
}

\begin{abstract}
Résumé En réanimation pédiatrique, $40 \%$ des décès surviennent à la suite d'une décision de limitation ou d'arrêt de traitement (LAT). Ces situations sont sources de questionnements éthiques complexes au sein de l'équipe soignante. La législation française et les recommandations des sociétés savantes donnent un cadre aux réanimateurs pédiatres pour les prises de décisions de LAT. Les enquêtes de pratiques nous montrent qu'ils se sont approprié certains éléments de la procédure collégiale comme la nécessité de la concertation pluriprofessionnelle, l'information et la communication avec les parents. Néanmoins, certains points tels que la présence du consultant, la réalité de la collégialité avec l'expression de toutes les personnes soignantes présentes sont encore insuffisamment appliqués. La place des parents dans les décisions doit être également réfléchie. La collaboration entre les équipes de réanimation pédiatrique et de médecine palliative est une possibilité pour améliorer sensiblement la qualité des soins et de l'accompagnement proposés. Cette collaboration élargit également les possibilités de la réflexion éthique nécessaire dans les situations de fin de vie complexes. L'intégration dans l'enseignement de la réanimation des principes de la médecine palliative est en cours de réflexion.
\end{abstract}

\footnotetext{
L. de Saint Blanquat $(\bowtie)$

Service de réanimation pédiatrique polyvalente,

hôpital universitaire Necker-Enfants-malades, AP-HP,

149, rue de Sèvres, F-75015 Paris, France

e-mail : laure.desaintblanquat@aphp.fr

M.-L. Viallard $(\bowtie)$

UF Douleur et médecine palliative périnatale, pédiatrique et adulte,

hôpital universitaire Necker-Enfants-malades, AP-HP,

149, rue de Sèvres, F-75015 Paris, France

e-mail : marcel-louis.viallard@aphp.fr
}

Laboratoire de recherche en éthique translationnelle, université Paris-Descartes, Sorbonne-Paris-Cité, 45, rue des Saints-Pères, F-75006 Paris, France
Mots clés Réanimation pédiatrique $\cdot$ Limitation et arrêt de traitement . Démarche palliative intégrée

Abstract In pediatric intensive care unit (PICU), $40 \%$ of deaths occur after a withholding or withdrawing life sustaining treatment (WLST) decision. These situations are often the source of ethical dilemmas among members of the health care team. French law and clinical guidelines established by academic societies provide a framework for PICU caregivers during WLST decisions. National surveys show that they have appropriated some of the collegial procedures like pluri-professional concertation, information and communication with the parents. Nevertheless, some points such as the presence of the consultant, the reality of the collegiality with each present person's expression are not routinely done. The parents' place during the decision making also must be thought. The collaboration PICU team and palliative medicine team appear as a possibility to improve yet the quality of the care and the accompanying which are proposed. Those collaborations also enlarge the possibility of ethical thinking needed during the end of life or a complex situation. Additional efforts in PICU professionals training are desirable as well as the development of a regular collaboration with palliative medicine professionals.

Keywords PICU · Withholding or withdrawing life sustaining treatment $\cdot$ Integrated palliative care

\section{Introduction}

La technicité et les performances des soins intensifs se sont améliorées au cours des 30 dernières années. En 2006, en France, la mortalité au cours d'un séjour en réanimation pédiatrique était de 7,3\% [1]. Dans de nombreuses situations, les moyens de suppléance, plus performants, permettent à l'enfant gravement malade de récupérer ad integrum ou à défaut de poursuivre un parcours de vie dans les meilleures conditions de « santé » possibles. 
Cependant, il existe des situations où la maladie causale continue d'évoluer, parfois aggravée par des complications secondaires aux thérapeutiques invasives de réanimation. La prise en charge doit alors être réfléchie pour éventuellement réorienter l'objectif des traitements et des soins assurés à l'enfant du curatif vers le palliatif. L'équipe soignante de réanimation peut alors décider de limiter ou d'arrêter un ou des traitements de suppléance vitale (limitation et arrêt des traitements [LAT]) en respectant une procédure collégiale. En France, $40 \%$ des décès en réanimation pédiatrique font suite à des décisions de LAT [1,2]. Le cadre juridique a évolué depuis 15 ans, il précise les modalités de prise de décision de LAT sans pour autant permettre de résoudre tous les dilemmes éthiques. Les résultats des enquêtes de pratique montrent l'émergence des situations cliniques qui suscitent de plus en plus le questionnement éthique au sein des équipes de réanimation. Dans ces situations complexes, l'intégration précoce d'une démarche palliative en réanimation pourrait permettre une amélioration de la prise en charge non seulement de l'enfant, de sa famille, mais également de l'équipe soignante.

Cet article envisage une mise au point sur l'évolution des réflexions éthiques dans les réanimations pédiatriques et l'intérêt d'une intégration d'une démarche palliative dans ce contexte particulier.

\section{Évolution de la législation française}

\section{Cadre juridique}

Tout en maintenant l'interdiction d'euthanasie et en réaffirmant le droit à l'accès aux soins palliatifs, trois grandes lois ont marqué l'évolution du droit des personnes malades et de la fin de vie. La loi du 4 mars 2002 consacre le principe d'autonomie du patient qui, après avoir reçu une information éclairée, a le droit de refuser un traitement [3]. La loi du 22 avril 2005 « dite Leonetti » autorise le médecin à limiter ou à arrêter un traitement dès lors qu'il est jugé inutile ou disproportionné chez un patient incapable d'exprimer sa volonté, à partir du moment où la décision fait suite à une procédure collégiale. Le statut de personne de confiance et les directives anticipées sont créés [4]. Enfin, la loi du 3 février 2016 « dite ClaeysLeonetti » renforce le poids de ces deux derniers dans la décision médicale. Elle donne le droit au patient atteint d'une affection grave et incurable et dont le pronostic vital est engagé à court terme à une sédation profonde et continue maintenue jusqu'au décès. Le médecin doit la mettre en place soit à la demande du patient en cas de souffrance réfractaire ou incontrôlable ou en cas de décision d'arrêt de traitement susceptible d'engager le pronostic vital à court terme et/ou d'être source de souffrance insupportable pour le patient, soit chez le patient inconscient lors de l'application d'un retrait ou d'une limitation d'un ou des traitements maintenant en vie artificiellement [5].

\section{Application de la loi à l'enfant et au nouveau-né}

Les textes réglementaires et législatifs au sujet de la fin de vie n'abordent pas spécifiquement les situations pédiatriques ou néonatales. Le mineur est considéré comme une personne vulnérable devant être protégée au même titre qu'un majeur incapable d'exprimer sa volonté. La loi s'applique donc à l'enfant et au nouveau-né. Les titulaires de l'autorité parentale, le plus souvent les parents, ont un devoir de protection (article 371-1 du Code civil). Pour cette raison, ils doivent être informés de la décision, et leur avis doit être sollicité. Le consentement du mineur doit être systématiquement obtenu s'il est apte à exprimer sa volonté et à participer à la décision. La décision est prise par le médecin référent qui en assume la responsabilité juridique.

\section{Recommandations des sociétés savantes}

En 1998, le Groupe francophone de réanimation et urgences pédiatriques (GFRUP) a entamé une réflexion pluridisciplinaire sur les LAT en réanimation pédiatrique, publiée en 2002 sous la forme d'un fascicule intitulé « LAT en réanimation pédiatrique; repères pour la pratique » (Tableau 1) [6]. En 2005, avant la promulgation de la loi « dite Leonetti », le GFRUP a écrit des recommandations visant à aider les professionnels de santé à la prise en charge les enfants dans ces situations complexes [7]. Trois repères éthiques - ne pas nuire, intérêt supérieur de l'enfant ou principe de bienfaisance et le principe d'autonomie — devant être déclinés vis-à-vis de l'enfant, de ses parents et de l'équipe soignante ont été définis ainsi qu'un certain nombre de termes pouvant être sources de confusion dans la littérature scientifique. Les recommandations concernaient les modalités du processus décisionnel, la mise en œuvre de la décision, les modalités d'application de la décision, l'accompagnement et le soutien des parents et de la famille après la mort de l'enfant. Les grandes lignes sont résumées dans le tableau 2. À la demande du GFRUP, un groupe de travail s'est constitué et travaille sur une actualisation de ces recommandations pour la pédiatrie au regard de l'évolution de la loi.

\section{Pratiques et les réflexions actuelles des équipes de réanimation pédiatrique}

\section{Fréquence du questionnement sur le bien-fondé des traitements et fréquence des décisions de LAT}

Depuis une quinzaine d'années, différentes études épidémiologiques conduites en France ont montré que le taux de décès par LAT restait stable, autour de $40 \%[1,2]$. 
Tableau 1 LAT, processus décisionnel de LAT : recommandations du GFRUP [11] / Withholding, withdrawing : GFRUP recommendations

\section{Processus décisionnel}

Questionnement sur l'utilité des traitements

Organisation d'une réunion de concertation

pluridisciplinaire associant l'ensemble des professionnels de santé en charge de l'enfant

Recueil préalable de l'avis des parents

Élaboration et prise d'une décision à la suite d'une discussion argumentative prenant en compte des facteurs médicaux et non médicaux, et respectant une éthique de communication, témoin d'une collégialité dans la discussion

Notification dans le dossier (noms des participants, décision et arguments)

Définition d'un nouveau projet de soins

\section{Mise en œuvre de la décision}

Respecter un délai de mise en œuvre

Information de l'enfant

Information des parents

Information du reste de l'équipe soignante

Information des équipes pouvant être amenées à prendre

en charge l'enfant après le séjour en réanimation

\section{Modalités d'application de la décision}

Favoriser la présence de proches

Modalités d'arrêt des traitements : présence du médecin, lieu, prévention de la douleur et de l'inconfort

Accompagnement et soutien après la mort de l'enfant

Soutien des familles : photographie de l'enfant, livret de deuil Soutien et formation des soignants : analyse de pratiques, soutien psychologique

Alors que la législation française a beaucoup évolué durant ces 15 dernières années, la stabilité du nombre de décès consécutifs à des LAT contraste avec les chiffres rapportés dans plusieurs études menées dans des réanimations pédiatriques américaines, australiennes ou en GrandeBretagne (respectivement 68, 74 et $65 \%$ ) [8-10].

À l'heure où la médecine devient de plus en plus performante, le questionnement du bien-fondé des traitements devrait se poser quotidiennement pour tous les patients en réanimation. L'implémentation d'un programme de communication et de formation au questionnement éthique pourrait favoriser l'émergence de ce questionnement et améliorer les pratiques de tous les services de réanimation [11].

\section{Modalités de prise de décision}

La loi et plusieurs décrets d'application ont certes précisé les grandes lignes du processus de prise de décision de LAT
Tableau 2 Tableau récapitulatif

1) Pour l'enfant comme pour l'adulte, la législation française et les recommandations des sociétés savantes encadrent la procédure collégiale de décision de LAT

2) Certains éléments de la procédure collégiale sont acquis, mais certains restent encore insuffisamment appliqués : présence du médecin consultant, réalité de la collégialité avec prise de parole des paramédicaux et intégration de leur position dans la décision

3) La place des parents dans le processus de décision a largement évolué durant ces 20 dernières années. Ils sont désormais presque toujours informés de la décision, et leur accord est recherché. Les équipes de réanimation doivent réfléchir à la gestion des situations de désaccord avec les parents à l'heure où la législation va dans le sens d'un renforcement de l'autorité parentale

4) L'intégration d'une démarche palliative dans la prise en charge en réanimation a montré de nombreux bénéfices pour l'enfant, ses parents et l'équipe soignante. Ces bénéfices sont d'autant plus importants que l'intégration de la médecine palliative dans la prise en charge est précoce. La participation d'une équipe mobile de médecine palliative à la stratégie de soins de l'enfant doit être plus fréquemment sollicitée

5) La formation médicale intègre désormais des possibilités de formation transversale en médecine palliative. Cet effort doit être maintenu et devrait contribuer à une amélioration de la prise en charge en réanimation pédiatrique

$[3,4,12,13]$. Mais prendre une décision de LAT de suppléance vitale est un exercice difficile en réanimation, car elle entraîne le décès presque certain et rapide de l'enfant. En effet, dans une enquête de pratique menée dans les réanimations pédiatriques françaises entre 2006 et 2007, lorsqu'une décision d'arrêt de traitement était prise, $74 \%$ des enfants décédaient au décours de l'application de la décision [1]. Oberender et Tibballs trouvent des résultats similaires en 2011 avec $78 \%$ des enfants qui décédaient après un arrêt des traitements dans un délai très rapide (médiane à 10 minutes avec un maximum de 6,4 heures) [14].

Plusieurs études ont mis en évidence une amélioration des pratiques, puisque plusieurs éléments de la procédure collégiale exigée par la loi sont désormais acquis dans les services de réanimation pédiatrique $[1,2]$. Dans l'enquête de pratique réalisée en 2006-2007, une réunion de délibération collégiale était organisée pour $84 \%$ des décisions. Alors qu'en 2000 seulement $46 \%$ des infirmières participaient aux réunions, ce chiffre atteignait $87 \%$ en 2007. De la même manière, le pourcentage de parents informés de la décision augmentait de moins de $20 \%$ en 2000 à $87 \%$ en 2007. En 2010, plus de $93 \%$ des médecins et des paramédicaux 
étaient convaincus de la nécessité de participation des paramédicaux à la réunion de délibération collégiale.

Toutefois, la confrontation des exigences de la loi à la réalité du terrain a fait émerger certaines difficultés, les principales étant la présence et le rôle du médecin consultant et l'exercice de la collégialité.

La présence d'un médecin consultant étranger à l'équipe prenant en charge le patient et sans lien hiérarchique est une obligation légale. Au regard de la loi, le médecin consultant assure la présence d'un tiers neutre et bienveillant pouvant éclairer le médecin dans sa prise de décision [15]. Cependant, les équipes de réanimation pédiatrique ne semblent pas être toutes persuadées de l'obligation et de l'intérêt de sa présence. En 2006, le médecin consultant ne participait qu'à seulement $50 \%$ des réunions [1]. En 2010, moins de la moitié des médecins et à peine un tiers des paramédicaux étaient toujours favorables à sa participation aux réunions de délibération pluriprofessionnelle [16]. La notion de médecin consultant semble être encore floue dans l'esprit des réanimateurs pédiatres sans que les raisons aient été identifiées. Le médecin consultant est souvent assimilé au «médecin expert » dont le rôle est sensiblement différent.

La nécessité d'intégrer des paramédicaux et notamment des infirmiers dans les discussions précédant la prise de décision est largement reconnue dans la littérature et correspond non seulement à leur souhait, mais également à celui des médecins [17]. La présence physique des paramédicaux aux réunions de délibération pluriprofessionnelle est d'ailleurs devenue au fil du temps une réalité dans les réanimations pédiatriques [1]. Cependant, dans une étude en 2010 sur le ressenti des soignants vis-à-vis des décisions de LAT en réanimation pédiatrique, seulement près d'un paramédical (infirmiers et aides-soignants) sur deux pensait qu'il avait la possibilité de s'exprimer lors de ces réunions, et $60 \%$ de ces mêmes soignants pensaient que leur avis n'était rarement ou jamais pris en compte [16]. Or, une délibération collégiale implique non seulement une présence physique, mais également une participation active de tous les acteurs de soins prenant en charge l'enfant.

Si la loi a inscrit cette obligation, elle n'en a pas précisé les modalités qui devraient permettre à chaque participant — notamment les paramédicaux — de s'exprimer. Il appartient aux professionnels de santé de réfléchir à des solutions permettant de rendre la collégialité réellement effective, alors que la formation médicale et paramédicale aborde très peu la communication interprofessionnelle et ses outils. Une réflexion pluriprofessionnelle réunissant médecins de spécialités différentes, psychologues, infirmière, sociologue visant à améliorer la collégialité en pédiatrie propose de mettre l'accent sur trois points : réunir l'ensemble des personnes concernées, obtenir l'expression de tous et s'assurer la compréhension de tous en limitant le pouvoir d'un discours exclusivement médical [18]. Un scé- nario « idéal » de réunion de délibération pluriprofessionnelle est proposé dans ce travail, détaillant les nécessités organisationnelles et le déroulement des différentes étapes, permettant à chaque service de s'approprier certaines suggestions. Ainsi, parvenir à rendre la collégialité réelle et opérante pour tous les professionnels est l'un des enjeux éthiques actuels en réanimation.

\section{Communication et place des parents dans les décisions de LAT}

Selon la loi française, l'enfant est représenté par ses parents (ou les titulaires de l'autorité parentale) qui ont un devoir de protection vis-à-vis de lui jusqu'à sa majorité. L'autorité parentale relevant du devoir, elle ne saurait être sacralisée puisqu'en cas de défaillance elle peut être retirée par la justice. À la différence de la médecine adulte où les interlocuteurs de l'équipe médicale sont devenus les proches plus que la famille au sens strict du terme, les réanimateurs pédiatres ont des interlocuteurs clairement identifiés.

En pédiatrie, les médecins ont longtemps adopté le modèle d'une médecine paternaliste vis-à-vis des parents. Il semblait impossible de demander à des parents de s'impliquer dans le processus de décision qui pouvait ou allait entraîner la mort de leur enfant. Cette pensée a largement été remise en question depuis une vingtaine d'années, et plusieurs études ont démontré que le partage d'une décision de LAT avec les parents était non seulement un souhait des parents, mais pouvait également avoir un effet bénéfique sur leur vécu de la situation et du décès éventuel de leur enfant [19-22]. Carnevale et al. ont montré que le niveau de culpabilité était indépendant de leur niveau d'implication dans la décision [23]. La loi de 2005 a inscrit l'obligation de recueillir l'avis des parents et de les informer de la décision [4]. Les recommandations du GFRUP ont adopté le principe de décision partagée.

«... Il est souhaitable que le médecin référent laisse aux parents le choix de leur niveau d'implication... Si les parents veulent avoir le sentiment de partager cette décision, le médecin référent doit savoir s'effacer pour les laisser prendre de l'autonomie et délivrer l'information de telle manière qu'ils puissent la recevoir comme une question » [6].

Exercice difficile dans la réalité : obtenir l'accord des parents en respectant leur choix et sans leur faire partager la responsabilité de la décision lorsqu'ils ne le souhaitent pas. La loi de 2016 a renforcé l'autonomie du patient visà-vis du pouvoir médical dans la prise en charge du patient adulte. Les implications pour les pédiatres sont difficiles à cerner. Certes, la loi de 2016 ne cite pas de renforcement de l'autorité parentale, mais en pédiatrie, l'enfant et ses parents sont sujets de soins, accentuant la complexité de la prise en 
charge. Le contexte de LAT est une situation à risque de conflit avec les parents. La volonté la plus fréquente des médecins est de ne « pas passer en force » pour des situations aussi complexes et dont les implications émotionnelles sont, pour les parents, très souvent dramatiques, s'accompagnant le plus souvent de la mort de leur enfant. L'équipe soignante peut se trouver confrontée à une tension éthique importante entre ce qu'elle estime être l'intérêt supérieur de l'enfant et le respect de la volonté des parents. Dans la plupart des cas, les médecins tentent d'éviter le conflit et temporisent pour ne pas compromettre l'alliance thérapeutique établie avec eux. Récemment, le jugement rendu par le tribunal administratif de Marseille puis l'arrêt du Conseil d'État ordonnant aux médecins de poursuivre les traitements de suppléance vitale allaient à l'encontre de ce qui était proposé par l'équipe soignante en charge de l'enfant (l'équipe proposant un retrait de la ventilation mécanique chez une enfant devenue tétraplégique, dépendante de la ventilation mécanique avec un état paucirelationnel à la suite d'une encéphalite sévère à entérovirus). L'esprit de la loi de 2016 et l'arrêt du Conseil d'État dans l'affaire de Marseille suggèrent un poids plus important donné à l'avis des parents. Une réflexion pluridisciplinaire associant différents professionnels de santé et juristes sur la place des parents visant l'élaboration de repères pour faciliter la gestion de conflits et pour aider le corps médical est en cours au niveau du Groupe de réflexion sur les aspects éthiques de la périnatologie (GRAEP).

\section{Sédation profonde et continue}

La loi impose, désormais, au médecin de mettre en place une sédation profonde et continue associée à une analgésie en cas de syndrome douloureux associé ou prévisible, lorsqu'un arrêt de traitement maintenant artificiellement en vie est décidé dans un contexte d'obstination déraisonnable. La décision de sédation doit être prise à la suite d'une procédure collégiale, pour un patient hors d'état d'exprimer sa volonté, et par conséquent, cela s'applique aussi pour un enfant. La sédation profonde et continue est définie comme l'effet obtenu par la prescription de moyens médicamenteux entraînant une altération profonde et persistante de la conscience, cette sédation devant être maintenue jusqu'au décès. Elle doit être associée systématiquement à une analgésie adaptée. Elle se différencie clairement des sédations habituellement mises en œuvre en réanimation. Le respect de la procédure collégiale est impératif, celle-ci étant 1'un des points centraux de différence avec une pratique euthanasique, car elle permet d'explorer de façon explicite en son sein les notions de temporalité, des moyens à utiliser pour sa mise en œuvre et l'intentionnalité. La réunion de délibération pluriprofessionnelle est un lieu de discussion, la décision étant de la seule responsabilité du médecin en charge du patient, les éléments de la délibération ayant pour vocation de lui apporter des éclairages complémentaires. Informer les parents est une obligation. Tous les arguments significatifs ayant émergé de la procédure collégiale, ceux sur lesquels le médecin en charge du patient s'est appuyé pour prendre sa décision et les éléments exprimés par les parents, doivent être colligés dans le dossier du patient. Dès lors qu'une sédation profonde, continue, maintenue jusqu'au décès est décidée et mise en œuvre, tous les traitements maintenant la vie artificielle doivent être arrêtés. La loi précise que la nutrition et l'hydratation artificielles sont des traitements et de ce fait peuvent également être arrêtées. Des recommandations récentes de la HAS sur la mise en place de ce type de sédation devraient accompagner les professionnels de santé pour s'approprier cette prise en charge [24]. Le travail d'actualisation des recommandations sous l'égide du GFRUP devrait également apporter des réponses.

\section{Intégration d'une démarche palliative dans la prise en charge des fins de vie en réanimation}

Depuis 2016, la loi impose la mise en place des soins palliatifs pour les patients qui font l'objet de décisions de LAT. Au-delà du contexte particulier de la LAT, la Société de réanimation de langue française (SRLF) a également souligné la nécessité de l'intégration d'une démarche palliative aux traitements actifs et l'importance de réduire au maximum les souffrances du patient [25].

Les soins palliatifs ne sont plus l'ultime recours avant la mort, ils doivent être intégrés précocement dans un projet thérapeutique modifié, adapté au patient en fin de vie ou à celui dont la vie est menacée. En médecine adulte, l'intérêt pour le patient, sa famille et l'équipe soignante d'une intégration de soins palliatifs est démontré par de nombreuses études colligées dans une revue de la littérature publiée en 2015 [26]. En pédiatrie, cette notion est également une réalité pratique [27]. Proposer une démarche palliative intégrée à la prise en charge ayant une visée curative précocement dans l'évolution d'une pathologie potentiellement létale permettrait d'anticiper d'une part les symptômes possibles lors des derniers moments de vie et leur prise en charge, d'autre part d'assurer un accompagnement aux entourages comme aux patients et de soutenir les équipes médicosoignantes en charge des patients [28].

En 2013, une étude rétrospective s'était intéressée aux caractéristiques du décès des enfants selon qu'ils avaient reçu des soins palliatifs ou non. Lorsqu'une réflexion palliative était intégrée, les enfants avaient une prise en charge moins agressive (ventilation mécanique, nutrition parentérale, chirurgie) et décédaient moins souvent dans le service de 
réanimation. [29]. Trois modèles d'incorporation des soins palliatifs en réanimation ont été décrits dans la littérature :

- consultatif où une équipe mobile de médecine palliative intervient auprès de patients identifiés à haut risque de décès, cette équipe assurant un suivi transversal du patient s'il sort de réanimation pour un autre service, le problème étant les critères permettant l'identification précoce de ces patients ;

- intégrés dans la prise en charge de la réanimation avec un screening quotidien des patients pouvant relever d'une démarche palliative et des protocoles de soins à visée de confort, le principal écueil étant l'indispensable formation des personnels médicaux et paramédicaux de la réanimation ;

- le modèle mixte associant les deux modèles précédents [30].

À partir des trois modèles d'incorporation, une revue de la littérature récente souligne les différents champs d'amélioration lors de l'incorporation des soins palliatifs en réanimation : prise en charge de la douleur, qualité de vie de l'enfant et de sa famille, communication, aide à la prise de décision, suivi de deuils des parents et de la fratrie. La prise en compte des soins palliatifs serait un indicateur de bonne qualité de soins [31].

En France, le délai entre la décision et sa mise en œuvre est de 48 heures [1]. La possibilité de préserver un temps entre le moment où la décision de LAT est prise et sa mise en œuvre est un élément qui pourrait favoriser d'une part l'acceptation pour les équipes médicosoignantes et d'autre part, pour les parents, « l'intériorisation » et la « maturation » de la réalité difficile à laquelle ils sont confrontés. Ce temps participe à éviter toute impression de précipitation et peut permettre de rendre possibles d'éventuels rites confessionnels ou familiaux, des temps d'échanges permettant d'exprimer les ressentis ou tout questionnement qui pourrait persister. L'intégration d'une prise en charge palliative pourrait permettre d'aider les équipes à accepter cette temporalité, peu habituelle en réanimation, qui permettrait également de mieux singulariser la situation.

La participation aux décisions de LAT, la prise en charge d'un enfant en fin de vie et son décès en réanimation pédiatrique sont sources de stress pour le personnel soignant [3234]. Jonas et Bogetz [35] ont mis en évidence le bénéfice que pouvaient apporter l'association d'une équipe de soins palliatifs à la prise de décision et la prise en charge d'un enfant en fin de vie et de sa famille. L'équipe de soins palliatifs peut être un intermédiaire très utile entre l'équipe soignante et la famille, s'assurant que chaque partie a bien été entendue. Elle peut se positionner comme tiers facilitant l'expression des émotions et les difficultés des soignants et de la famille.

$\mathrm{Au}$ moment du décès de l'enfant, les besoins des parents ont été largement étudiés. Une revue récente de la littérature a souligné l'importance de leur redonner le rôle de parents. [36]. Ils appréhendent le moment du « mourir» sans souvent oser demander ce qu'il va se passer. L'équipe de soins palliatifs peut aider à donner des explications sur les manifestations attendues comme les gasp et leur prise en charge.

La collaboration avec les équipes de médecine palliative adulte ou pédiatrique ou mixte est considérée par plusieurs auteurs comme pouvant être source d'amélioration de la qualité des soins délivrés à l'enfant, de l'accompagnement des parents et des entourages élargis, et de la satisfaction des familles quant à la prise en charge de la fin de vie de l'enfant [37,38].

Enfin, l'intégration précoce d'une équipe de médecine palliative dans la prise en charge a un intérêt certain en cas de survie de l'enfant [26]. En effet, dans l'étude PEDIALAT, $24 \%$ des enfants pour lesquels une décision de LAT avait été prise sortaient vivants de réanimation. Les résultats préliminaires pour 57 enfants montrent que $92 \%$ des médecins en charge de ces enfants avaient connaissance de la décision de LAT. Et pourtant, à partir d'un scénario fictif, une nouvelle admission en réanimation serait demandée pour $47 \%$ des enfants. Grâce à sa transversalité, une équipe mobile de médecine palliative pourrait permettre une nouvelle discussion du projet le plus raisonnable pour l'enfant au vu de son évolution clinique à distance de la réanimation [39].

\section{Perspectives}

Le besoin de formation spécifique en médecine palliative pour les professionnels médecins, paramédicaux et autres intervenants comme les psychologues ou les kinésithérapeutes par exemple est clairement identifié comme nécessaire pour bien comprendre que la démarche palliative ne se limite pas aux seuls derniers moments de vie (soins palliatifs terminaux) $[40,41]$.

Le développement de l'enseignement de la médecine palliative, son intégration dans les différentes filières comme celles de la pédiatrie ou de l'anesthésie-réanimation [42] et la création d'une unité d'enseignement transversale portant sur les démarches palliatives et s'adressant à tous les professionnels de santé au décours des premier ou deuxième cycles des études des professionnels de santé [43] sont des innovations pertinentes qui devraient favoriser la compréhension des enjeux et le bénéfice de l'intégration précoce d'une démarche palliative dans la prise en charge des patients gravement malades.

Il est probablement souhaitable de mieux valoriser l'objectif du confort de l'enfant et de ses proches dans sa prise en charge globale associant le cure et le care qui sont les deux objectifs de la prise en charge en réanimation. 


\section{Conclusion}

Les évolutions de la réflexion éthique en réanimation pédiatrique se poursuivent au fil de l'émergence de situations de plus en plus complexes. Les pratiques se sont améliorées au fil du temps, et certaines exigences de la législation sont désormais acquises par les professionnels de santé. Cependant, certains éléments comme la réalité de la collégialité, la place des parents et la résolution de conflits nécessitent une réflexion plus approfondie afin d'être pleinement intégrés dans la pratique quotidienne. L'intégration précoce d'une démarche palliative en réanimation est une piste d'amélioration pour la prise en charge de l'enfant, de sa famille et de l'équipe soignante lors de ces situations complexes. En outre, si la majorité des décisions de LAT aboutissent à la mort de l'enfant, certains d'entre eux sont susceptibles de survivre, et une démarche palliative intégrée pourrait faciliter la mise en place d'une prise en charge appropriée lors de la sortie de réanimation pour l'enfant. L'enseignement de la médecine palliative est en cours de réflexion et devrait favoriser la collaboration entre les différentes équipes.

Liens d'intérêts : les auteurs déclarent ne pas avoir de lien d'intérêt.

\section{Références}

1. Cremer R, Hubert P, Grandbastien B, Moutel G, Leclerc F; GFRUP's study group on forgoing treatments, (2011) Prevalence of questioning regarding life-sustaining treatment and time utilisation by forgoing treatment in francophone PICUs. Intensive Care Med 37: 1648-1655

2. Devictor DJ, Nguyen DT; Groupe francophone de réanimation et d'urgences pédiatriques, (2001) Forgoing life-sustaining treatments: how the decision is made in French pediatric intensive care units. Crit Care Med 29: 1356-1359

3. Loi (française) du 4 mars 2002 relative aux droits des malades et à la qualité du système de santé. Journal officiel de la République française du 5 mars 2002

4. Loi (française) $n^{\circ}$ 2005-370 du 22 avril 2005 relative aux droits des malades et à la fin de vie. Journal officiel de la République française du 23 avril 2005

5. Loi (française) $n^{\circ} 2016-87$ du 2 février 2016 créant de nouveaux droits en faveur des malades et des personnes en fin de vie. Journal officiel de la République française du 3 février 2016

6. Groupe francophone de réanimation et d'urgences pédiatriques (GFRUP), (2002) Limitation ou arrêt des traitements en réanimation pédiatrique : repères pour la pratique. Fondation de France, Paris, $155 \mathrm{p}$

7. Hubert P, Canouï P, Cremer R, Leclerc F, (2005) Withholding or withdrawing life saving treatment in pediatric intensive care unit: GFRUP guidelines. Arch Pediatr 12: 1501-1508

8. Meert KL, Keele L, Morrison W, Berg RA, Dalton H, Newth CJ, Harrison R, Wessel DL, Shanley T, Carcillo J, Clark A, Holubkov R, Jenkins TL, Doctor A, Dean JM, Pollack M; Eunice Kennedy Shriver National Institute of Child Health and Human Development Collaborative End-of-Life Pediatric Critical Care
Research Network, (2015) Practices among tertiary care PICUs in the United States: a multicenter study. Pediatr Crit Care Med 16: e231-238

9. Moore P, Kerridge I, Gillis J, Jacobe S, Isaacs D, (2008) Withdrawal and limitation of life-sustaining treatments in a paediatric intensive care unit and review of the literature. J Paediatr Child Health 44: 404-408

10. Sands R, Manning JC, Vyas H, Rashid A, (2009) Characteristics of deaths in paediatric intensive care: a 10-year study. Nurs Crit Care 14: 235-240

11. Chastrusse M, Fulgencio JP, Julien F, Naudin B, Argo V, Bonnet F, Ferrand E, (2012) Loi n ${ }^{\circ}$ 2005-370 du 22 avril 2005 relative aux droits des malades et à la fin de vie : amélioration du processus de décision de limitation et d'arrêt des traitements au moyen d'un programme pédagogique. Étude monocentrique prospective et rétrospective pilote. Presse Med 41: e539-546

12. Décret $n^{\circ} 2006-119$ du 6 février 2006 relatif aux directives anticipées prévues par la loi $\mathrm{n}^{\mathrm{o}} 2005-370$ du 22 avril 2005 relative aux droits des malades et à la fin de vie et modifiant le Code de la santé publique (dispositions réglementaires). Journal officiel de la République française du 7 février 2006

13. Décret $n^{\circ} 2016-1066$ du 3 août 2016 (France) modifiant le Code de déontologie médicale et relatif aux procédures collégiales et au recours à la sédation profonde et continue jusqu'au décès prévus par la loi $\mathrm{n}^{\circ}$ 2016-87 du 2 février 2016 créant de nouveaux droits en faveur des malades et des personnes en fin de vie. Journal officiel de la République française du 5 août 2016

14. Oberender F, Tibballs J, (2011) Withdrawal of life-support in paediatric intensive care - a study time intervals betwwen discussion, decision and death. BMC Pediatr 11: 39

15. Cremer R, Fayoux P, Guimber D, Joriot S, Laffargue A, Lervat C, Matthews A, Mention K, Sfeir R, Storme L, Thomas D, Thumerelle C, Vandoolaeghe S; le groupe de travail de l'EEHU de Lille, (2012) The consulting physician for withdrawal of lifesustaining treatments in children. Arch Pediatr 19: 856-862

16. de Saint Blanquat L, Cremer R, Elie C, Lesage F, Dupic L, Hubert $\mathrm{P}$; pour le Groupe francophone de réanimation et d'urgences pédiatriques (GFRUP), (2014) French law related to patient's rights and end of life: pediatric intensive care unit's health professionals' opinions. Arch Pediatr 21: 34-43

17. Flannery L, Ramjan LM, Peters K, (2016) End-of-life decisions in the intensive care unit (ICU) - exploring the experiences of ICU nurses and doctors. A critical literature review. Aust Crit Care 29: 97-103

18. Cremer R, Lervat C, Laffargue A, Le Cunff J, Joriot S, Minnaert C, Cuisset JM, Mention K, Thomas D, Guimber D, Matthews A, Fayoux P, Storme L, Vandoolaeghe S; groupe de travail de l'EEHU de Lille, (2015) Comment organiser la délibération collégiale pour limiter ou arrêter les traitements en pédiatrie ? Arch Pediatr 22: 1119-1128

19. Meert KL, Thurston CS, BrillerSH, (2005) The spiritual needs of parents at the time of their child's death in the pediatric intensive care unit and during bereavement: a qualitative study. Pediatr Crit Care Med 6: 420-427

20. Meyer EC, Burns JP, Griffith JL, Truog RD, (2002) Parental perspectives on end-of-life care in the pediatric intensive care unit. Crit Care Med 30: 226-231

21. McGraw SA, Truog RD, Solomon MZ, Cohen-Bearak A, Sellers DE, Meyer EC, (2012) "I was able to still be her mom" - parenting at end of life in the pediatric intensive care unit. Pediatr Crit Care Med 13: e350-356

22. Caeymaex L, Jousselme C, Vasilescu C, Danan C, Falissard B, Bourrat MM, Garel M, Speranza M, (2013) Perceived role in end-of-life decision making in the NICU affects long-term parental grief response. Arch Dis Child Fetal Neonatal Ed 98: F26-31 
23. Carnevale FA, Canouï P, Cremer R, Farrell C, Doussau A, Seguin MJ, Hubert P, Leclerc F, Lacroix J, (2007) Parental involvement in treatment decisions regarding their critically ill child: a comparative study of France and Quebec. Pediatr Crit Care Med 8: 337-342

24. HAS, (2018) Guide parcours de soins. Comment mettre en œuvre une sédation profonde et continue maintenue jusqu'au décès ? Consultable à l'adresse url : https://www.has-sante.fr/portail/ jcms/c_2832000/fr/comment-mettre-en-oeuvre-une-sedation-profonde-et-continue-maintenue-jusqu-au-deces?

25. Boyer A, Eon B, Quentin B, Blondiaux I, Bordet F, Dray S, JarsGuincestre MC, Noizet O, Gonzalez F, Pillot J, Rigaud JP, Rolando S, Vanderlinden T, Reignier $\mathrm{J}$; pour la commission d'éthique de la Société de réanimation de langue française, (2016) Que change la loi Claeys-Leonetti pour les réanimateurs ? Réanimation 25: 419-425

26. Khandelwal N, Kross EK, Engelberg RA, CoeNB, Long AC, Curtis JR, (2015) Estimating the effect of palliative care interventions and advance care planning on ICU utilization: a systematic review. Crit Care Med 43: 1102-1111

27. Viallard ML, (2009) Apports possibles d'une équipe mobile d'accompagnement et de soins palliatifs dans les situations de limitation et d'arrêt de traitement actif. Médecine palliative 8: 3-6

28. Blanchet V, Viallard ML, (2012) De l'accompagnement des mourants à la médecine palliative en passant par les soins palliatifs. Med Palliat 11: 266-270

29. Keele L, Keenan HT, Sheetz J, Bratton SL, (2013) Differences in characteristics of dying children who receive and do not receive palliative care. Pediactrics 132: 72-78

30. Nelson JE, Curtis JR, Mulkerin C, Campbell M, Lustbader DR, Mosenthal AC, Puntillo K, Ray DE, Bassett R, Boss RD, Brasel KJ, Frontera JA, Hays RM, Weissman DE; Improving Palliative Care in the ICU (IPAL-ICU) Project Advisory Board, (2013) Choosing and using screening criteria for palliative care consultation in the ICU: a report from the Improving Palliative Care in the ICU (IPAL-ICU) Advisory Board. Crit Care Med 41: 2318-2327

31. Boss R, Nelson J, Weissman D, Campbell M, Curtis R, Frontera J, Gabriel M, Lustbader D, Mosenthal A, Mulkerin C, Puntillo K, Ray D, Bassett R, Brasel K, Hays R, (2014) Integrating palliative care into the PICU: a report from the Improving Palliative Care in the ICU Advisory Board. Pediatr Crit Care Med 15: 762-767

32. Klein SM, (2009) Moral distress in pediatric palliative care: a case study. J Pain Symptom Manage 38: 157-160
33. Meltzer LS, Huckabay LM, (2004) Critical care nurses' perceptions of futile care and its effect on burnout. Am J Crit Care 13: 202-208

34. Wagner C, (2015) Moral distress as a contributor to nurse burnout. Am J Nurs 115: 11

35. Jonas D, Bogetz JF, (2016) Identifying the deliberate prevention and intervention strategies of pediatric palliative care teams supporting providers during times of staff distress. J Palliat Med 19: 679-683

36. Butler AE, Hall H, Willetts G, Copnell B, (2015) Family experience and PICU death: a meta-synthesis pediatrics. Pediactrics 136: e961-973

37. Doorenbos A, Lindhorst T, Starks H, Aisenberg E, Curtis JR, Hays R, (2012) Palliative care in the pediatric ICU: challenges and opportunities for family-centered practice. Soc Work End Life Palliat Care 8: 297-315

38. Lewis-Newby M, Curtis JR, Martin DP, Engelberg RA, (2011) Measuring family satisfaction with care and quality of dying in the intensive care unit: does patient age matter? J Palliat Med 14: $1284-1290$

39. Cremer R, Binoche A, Le Reun C, Hubert P; pour le « GFRUP's study group on forgoing treatments », (2014) Devenir des enfants quittant la réanimation après une décision de limitation ou d'arrêt des traitements. État des connaissances en France. Reanimation 23: $409-413$

40. Truog RD, Campbell ML, Curtis JR, Haas CE, Luce JM, Rubenfeld GD, Rushton CH, Kaufman DC; American Academy of Critical Care Medicine, (2008) Recommendations for end-of-life care in the intensive care unit: a consensus statement by the American College of Critical Care Medicine. Crit Care Med 36: 953-963

41. Mularski RA, Puntillo K, Varkey B, Erstad BL, Grap MJ, Gilbert HC, Li D, Medina J, Pasero C, Sessler CN, (2009) Pain management within the palliative and end-of-life care experience in the ICU. Chest 135: 1360-1369

42. Collège des enseignants de médecine intensive réanimation. Le DESMIR. Consultable à l'adresse url : http://www.cnerea.fr/fr/ le-desmir.html (consulté le 15 juillet 2017)

43. Plan national 2015-2018 pour le développement des soins palliatifs et l'accompagnement en fin de vie. Consultable à l'adresse url : http://solidarites-sante.gouv.fr/IMG/pdf/031215_-_plabe56. pdf (consulté le 15 juillet 2017) 